\title{
Rate and determinants of non-adherence to a gluten-free diet and nutritional status assessment in children and adolescents with celiac disease in a tertiary Brazilian referral center: a cross-sectional and retrospective study
}

Maraci Rodrigues $^{* *}$, Glauce Hiromi Yonamine ${ }^{2}$ and Carla Aline Fernandes Satiro ${ }^{2}$

\begin{abstract}
Background: Compliance with a gluten-free diet (GFD) is difficult at all ages but particularly for teenagers due to social, cultural, economic, and practical pressures. The multidisciplinary team responsible for the treatment of patients with celiac disease and give support to their parents plays a special role on strengthening GFD and assessing the nutritional and physical health.

Methods: A cross-sectional and retrospective study including patients under 20 years of age, with biopsyconfirmed CD, followed regularly at the Department of Pediatrics, Division of Gastroenterology, Hospital das Clínicas, University of Sao Paulo, School of Medicine, Sao Paulo, Brazil, were surveyed using a questionnaire and serologic test applied between November 2011 and February 2012. A retrospective chart review of these patients was performed to collect the anthropometric data along with the results of the serologic test performed at the time of diagnosis and after at least 1 year of treatment with a GFD.

Results: We evaluated 35 patients aged between 2.4 and 19.9 years. Of these $68.6 \%$ were female, $88.6 \%$ had the typical form of the disease and $51.4 \%$ had other comorbidities. The mean age at diagnosis was 5.4 years. Despite dietary guidance, $20 \%$ reported non-adherence to the diet. Most children recovered the weight and height deficit after 5 years of treatment, and in some children, excessive weight gain became a concern.

Conclusion: The majority of transgressions occurred intentionally at home or at parties. There was a risk of excessive weight gain, especially in the first two years of treatment. More alternatives and easier access to low cost gluten-free foods, increasing the discussion about the benefits of adhering to a GFD among patients, families, and the general population, besides the acquisition of self-management skills, are crucial to fostering independent children and adolescents who have the knowledge and tools to manage life with CD.
\end{abstract}

Keywords: Celiac disease, Nutritional status, Gluten-free diet

\footnotetext{
* Correspondence: maraci@uol.com.br

'Department of Gastroenterology, Hospital das Clínicas, School of Medicine,

University of Sao Paulo (SMUSP), Av. Dr Eneas de Carvalho Aguiar, 255,

05403-000, Sao Paulo, Brazil

Full list of author information is available at the end of the article
} 


\section{Background}

Celiac disease is now defined as a genetically predisposed systemic autoimmune condition characterized by the presence of a variable combination of glutendependent enteropathy, other clinical manifestations, and CD-specific antibodies such as anti-tissue transglutaminase antibodies 2 (TG2-IgA and TG2-IgG) and anti-endomysial antibodies (EMAs) [1] . The disease occurs in individuals carrying the HLA class II DQ2 or DQ8 allelic variants haplotype and is characterized by an inflammatory enteropathy with various degrees of severity, and a wide range of gastrointestinal and extraintestinal symptoms and signs [1]. CD can reach far beyond the intestine and has been associated with many nongastrointestinal signs and symptoms such as dermatitis herpetiformis, short stature, osteoporosis, iron deficiency anemia, arthritis, headaches, fatigue, liver function abnormalities, myalgias, adverse pregnancy outcomes, tooth enamel defects, and others [2]. A strong association with autoimmune conditions such as type 1 diabetes mellitus has been observed, and it has been linked with some congenital disorders, such as IgA deficiency and Down syndrome [2]. This pathology is considered a major public health problem because of its high prevalence worldwide. Studies from the United States and Europe estimated that the mean prevalence of CD in the general population is approximately $1 \%$, with some regional differences. A similar disease prevalence has been found in other countries mostly populated by European descendants [3].

In Brazil, the prevalence of biopsy-confirmed CD in apparently healthy volunteer blood donors ranged from 1:214 to 1:286 in Sao Paulo city $[4,5] 1: 273$ in Sao Paulo countryside [6], 1:417 in southern Brazil [7] and 1:681 in Brasilia [8] . These preliminary results support the view that $C D$ is not a rare disease in Brazil.

Currently, the treatment of CD is based on lifelong exclusion of wheat, rye and barley [9], oats (because in Brazil oats may be contaminated with gluten), and other gluten-containing food products from the diet.

Adherence to a GFD is crucial not only for intestinal mucosal recovery and alleviation of symptoms, but also for the prevention of complications such as anemia, osteoporosis fractures and small-bowel lymphoma [9].

Compliance with a GFD is difficult at all ages but particularly for teenagers due to social, cultural, economic, and practical pressures. At this age group, the diet may be a psychological and social challenge. Occasions such as birthday parties, sleepovers, eating out, and even snack time at school can be difficult to be managed. A survey among college students with $\mathrm{CD}$ revealed that they were motivated to adhere to the diet but they experienced challenges related to dining services and social meetings [10].
The strategies required for a GFD to be successful must include education for the patient, family and all those involved with the patient (e.g. healthcare team and school) regarding product ingredients, labeling all gluten-free flours, having snacks on hand at school or work, and bringing gluten-free foods to social events [11]. With regard to parental knowledge, there is the need for clinicians and healthcare professionals to provide better education and more efficient counseling about gluten-free products for parents [12].

Although evidence-based recommendations for followup of pediatric patients with CD have not yet been established, some experts advise a yearly follow-up visit and monitoring of dietary compliance, with evaluation of the improvement in symptoms and signs, and analysis for biochemical and serologic markers of CD and screening for associated autoimmune thyroiditis [13].

Therefore, the aims of this study were to examine the rate and determinants of non-adherence to a GFD, as well as the dietary habits and nutritional status of children and adolescents diagnosed with $\mathrm{CD}$ at a tertiary referral center.

\section{Methods}

This was a cross-sectional and retrospective study, and included 35 patients with CD who attended a medical consultation, conducted in the Pediatric Gastroenterology Outpatient Clinic of the Department of Pediatrics, SMUSP, Sao Paulo, Brazil, between November 2011 and February 2012. These patients were children and adolescents with confirmed diagnosis according to the CD consensus of 2012 [14]:

1.Typical or atypical manifestations or belonging to a group at risk for $\mathrm{CD}$;

2.Positive serology (TG2 IgA, EMAs, or TG2 IgG if hypogammaglobulinemia IgA was diagnosed) or presence of human histocompatibility antigen genes (HLA) class II DQ2 or DQ8 allelic variants;

3. Confirmation of the diagnosis by duodenal biopsy, from the bulb (at least one biopsy) and from the second and third portion of the duodenum (at least four biopsies), and grading according to marsh-Oberhuber [15].

The celiac follow-up visits were defined as attending a medical appointment that addressed CD and documented symptoms associated with CD, GFD compliance, and/or CD serologies.

The medical team included pediatric gastroenterologists, nutritionists and social assistants to assess each child and teenage carrier of $C D$, and their parents, in relation to symptoms, nutritional status, education, adherence and social difficulties associated with adhering to a GFD.

Patients younger than 1 year or older than 20 years and those who did not attend the consultation and/or 
with whom contact was not possible because of obsolete phone numbers were excluded. Two patients did not agree to participate in the study.

Data were collected using a questionnaire constructed for this study comprising two open questions on social events and difficulties with the GFD, and closed questions about food routine (home, school, parties, travel), awareness, adherence and difficulties regarding the GFD.

This questionnaire was applied by trained professionals. Parents of patients younger than 13 years of age were instructed to complete the survey with their child. Adolescents older than 13 years of age were asked to complete the survey themselves, during the appointment. The questionnaire are presented in Additional file 1.

Additional information was obtained from medical records, including associated diseases, laboratory tests and anthropometric assessment at the time of diagnosis, 1, 2 and 5 years after diagnosis.

The anthropometric data of weight and height were measured during the medical consultation, on the day of application of the questionnaire. Weight was measured on digital scales, with accuracy to $100 \mathrm{~g}$, while the patient was barefoot and in his underwear. Height was measured using a horizontal stadiometer in children under 2 years of age and a vertical stadiometer in children above this age.

The anthropometric data of weight, height and body mass index (BMI) were expressed as $\mathrm{Z}$ scores using the World Health Organization (WHO) multicentre growth reference study group [16].

The laboratory tests were performed according to the reference laboratory of SMUSP.

\section{Ethics approval and consent to participate}

This project was approved by the Research Ethics Committee (REC) and the Ethics Committee for review of research projects (CAPPesq) under the number 829/11.

Patients (above 16 years of age), parents or legal guardians (in the case of children under 16) only participated in the study after signing the informed consent, which provided information on the goals of the study and other relevant information.

Permission to conduct the study was sought from relevant ethical committees at Department of Pediatric, Instituto da Criança, Hospital das Clinicas, São Paulo, Brazil. All patients were entered into the study after a written informed consent, either given by the patients themselves or their guardians in the case where patients were not able to.

\section{Statistics}

All data were coded in numerical form. The data tabulation and analysis were performed using Microsoft Excel, Epi Info, version 6.04, and the Statistical
Package for the Social Sciences, version 13.0 (SPSS Inc. Chicago, IL, USA).

Fisher's exact test was the statistical test used to determine if there were nonrandom associations between two categorical variables, and McNemar's test was used to compare paired proportions. Significance was defined as $P<0.05$.

\section{Results}

\section{Characterization of the population}

Were included a total of 35 patients, aged from 2.4 to 19.9 years (mean: 10.77, standard deviation: 4.16 and median: 11 years); $68.6 \%$ were female. In most cases (51.35\%), the questionnaires were answered by the patient's mother (Table 1).

Patients or their family considered their health to be good, very good or great (91.4\%) when compared to other people of the same age without the disease.

About $51.4 \%$ of respondents had another disease besides $\mathrm{CD}$, the most cited were: diabetes mellitus type 1 $23 \%(n=8)$, asthma $14 \%(n=5)$, and rhinitis and epilepsy $11.5 \%$ (both with four cases).

The majority of patients (91.4\%) did not have knowledge about CD before diagnosis, and 93.8\% reported that they had been suffering from symptoms characteristic of the disease (mainly diarrhea) for about 23.6 months before diagnosis (DP: 24 months).

\section{Dietary treatment}

Most patients consumed meat, milk, fruit and vegetables on a daily basis, as per Table 2 .

When at school, $48 \%(n=24)$ of the patients brought a snack from home, 18\% $(n=9)$ bought lunch. When the option was to purchase food at school, the favorites were: cheese bread (25.7\%),wich is very popular in Brazil and it is made with cassava flour and cheese, snacks (22.9\%), soda (14.3\%); and $69.2 \%$ did not receive GFD at school.

All patients were instructed to follow a restricted diet, but $20 \%(n=7)$ reported that they did not avoid gluten at all times. The latter presented a positive EMAs result.

Factors cited as justifications for transgressions were the high cost of a GFD $(n=2 ; 15.4 \%)$, the low acceptance of these foods $(n=2 ; 15.4 \%)$, lack of choice $(n=1$; $7.7 \%)$, visiting friends $(\mathrm{n}=1 ; 7.7 \%)$, and voluntary choice $(n=7 ; 53.8 \%)$.

Transgressions occurred at parties $(n=12 ; 23.5 \%)$, home $(n=11 ; 21.6 \%)$, school $(n=10 ; 19.6 \%)$, while shopping $(n=8 ; 15.7 \%)$, with friends $(\mathrm{n}=7 ; 13.7 \%)$, and while traveling $(n=3 ; 5.9 \%)$. A total of $51.9 \%$ claimed that the transgressions were intentional and $63.3 \%$ reported having symptoms after the transgressions, primarily diarrhea (41.4\%) and abdominal pain (41.4\%). 
Table 1 Characteristics of the population

\begin{tabular}{|c|c|c|}
\hline Features & $\mathrm{N}$ & $\%$ \\
\hline \multicolumn{3}{|l|}{ Gender } \\
\hline Boys & 24 & 68.6 \\
\hline Girls & 11 & 31.4 \\
\hline \multicolumn{3}{|l|}{ Age group } \\
\hline $2-5$ years & 5 & 14.3 \\
\hline $5-12$ years & 15 & 42.9 \\
\hline$>12<19.9$ years & 15 & 42.9 \\
\hline \multicolumn{3}{|l|}{ Manifestation of the disease } \\
\hline Typical & 31 & 88.6 \\
\hline Atypical & 4 & 11.4 \\
\hline \multicolumn{3}{|l|}{ Age at diagnosis } \\
\hline $0-2$ years & 8 & 22.9 \\
\hline $2-5$ years & 9 & 25.7 \\
\hline $5-12$ years & 14 & 40 \\
\hline$>12$ years & 0 & 0 \\
\hline No response & 4 & 11.4 \\
\hline \multicolumn{3}{|l|}{ Time of disease } \\
\hline $0-2$ years & 8 & 22.9 \\
\hline $2-4$ years & 5 & 14.3 \\
\hline $4-6$ years & 4 & 11.4 \\
\hline $6-8$ years & 4 & 11.4 \\
\hline $8-10$ years & 3 & 8.6 \\
\hline$>10$ years & 6 & 17.1 \\
\hline No response & 5 & 14.3 \\
\hline \multicolumn{3}{|l|}{ Monthly income } \\
\hline Up to 1 minimum wage & 9 & 25.7 \\
\hline Up to 2 minimum wage & 13 & 37.1 \\
\hline Up to 3 minimum wage & 7 & 20 \\
\hline$>3$ minimum wage & 6 & 17.1 \\
\hline \multicolumn{3}{|c|}{ Number of residents in the house } \\
\hline $1-3$ & 8 & 22.9 \\
\hline $4-6$ & 25 & 71.4 \\
\hline$>7$ & 1 & 2.9 \\
\hline No response & 1 & 2.9 \\
\hline
\end{tabular}

Table 2 Food profile of patients with celiac disease

\begin{tabular}{lllll}
\hline & Meat & Milk & Fruit & $\begin{array}{l}\text { Greens and } \\
\text { vegetables }\end{array}$ \\
\hline Daily & $62.9 \%$ & $91.4 \%$ & $62.9 \%$ & $54.3 \%$ \\
2 to 4 times a week & $37.1 \%$ & $8.6 \%$ & $31.4 \%$ & $37.1 \%$ \\
Does not consume & - & & $5.7 \%$ & $8.6 \%$ \\
\hline
\end{tabular}

The frequency of flatulence was higher in the group that did not strictly adhere to the diet $(p=0.083)$.

When asked about their behavior at parties and social events, the majority reported taking food from home to avoid eating food containing gluten, while $62.8 \%$ of patients stated that they never attended events due to their disease. See Table 3.

Gluten-free recipes were prepared by $88.6 \%$ of the patients and/or their guardians, and $41.9 \%$ prepared special recipes three times a week. The main recipes with satisfactory sensory characteristics, according to the patients, were: cakes $(80 \%)$ and pies (20\%). Bread (20\%) and pancakes $(8.57 \%)$ were considered to have less satisfactory sensory characteristics. The flours mostly used as a substitute for gluten were: corn starch, corn flour and potato starch. All patients reported buying gluten-free foods in conventional supermarkets $(n=30)$ and specialist shops $(n=26)$.

Meals outside the home were eaten a few times per month by $45.7 \%$ of the patients, mainly in restaurants and coffee shops.

When asked about difficulties related to adhering to the treatment of celiac disease, $37.1 \%$ had no difficulty, $25.7 \%$ little difficulty, and 20\% average difficulty, while $17.1 \%$ found it very difficult. The majority of patients $(74.3 \%)$ were able to talk with ease about celiac disease, but a significant percentage, either disliked or were ashamed of talking about it, $17.1 \%$ and $14.3 \%$ respectively. All claimed to know about the risks of nonadherence to the diet and to know that dietary treatment would be lifelong.

There was no association between diet transgression and gender form of presentation of the disease, eating habits, behaviors in relation to food in school and social events or knowledge about the disease.

\section{Nutritional evaluation}

Out of the 35 patients, 27 had anthropometric data of diagnosis, enabling an assessment of nutritional evolution. The remaining patients were diagnosed in another service or did not have this data in their chart.

At the time of diagnosis 4 children were classified as having a nutritional deficit according to their BMI/age, either being underweight or extremely underweight but

Table 3 Behavior of patients with celiac disease at parties and social events

\begin{tabular}{ll}
\hline Behavior at parties & $\%$ \\
\hline Takes food from home & $65.7 \%$ \\
Ashamed and does not attend & $11.4 \%$ \\
Only eats permitted foods & $5.7 \%$ \\
Does not eat & $8.6 \%$ \\
Infringes the GFD & $5.7 \%$ \\
\hline
\end{tabular}


this index improved considerably during treatment; no child had a weight deficit at the end of the study.

In the same way, when the children were evaluated in accordance with their stature/age i.e. the number of children with a height deficit decreased during treatment.

The number of children who were overweight or obese increased throughout the follow-up of these patients. See Tables 4 and 5.

There was no difference in the nutritional parameters (BMI/age and stature/age) between patients who reported transgression of the diet and those who demonstrated adherence to treatment.

It was also assessed the laboratory tests of these patients, and the main change was the EMAs, with about 28 patients with a positive result. The results of this blood tests were not statistically different over time.

\section{Discussion}

This study demonstrated that $20 \%$ of evaluated patients did not adhere completely to the GFD. The reasons for transgressions were related to the GF products (high cost, low palatability and lack of availability), social events with friends and voluntary choice. Surprisingly, the nutritional parameters were not influenced by the adherence to the diet. Probably, the transgression of the GFD was not high enough to compromise the weight gain and growth of the patients.

Our sample was small compared with some previous studies [12, 17-23], but larger than in others [24, 25], because our study was conducted in a single center and included only patients with regular outpatient gastroenterologist visits in the period between November 2011 and February 2012.

The majority of our patients were women (68.6\%), which was consistent with other studies, in which a slight predominance of females was observed [12, 17-20, 22, 24, 26].

The mean age of the 35 children included in this study was 10.77 years and the median was 11 years; they were older than in some studies $[17,22,25]$ and younger than in other studies [12, 19, 20, 24].

In our study, the genre and clinical presentation of celiac disease at diagnosis had no effect on adherence. This is consistent with several other studies [12, 18, 22], but contrasts with others [27], in which adherence was better in girls than in boys.

In our study, the adherence of adolescents (older than 13 years of age) to a GFD was particularly low while dining at restaurants, maybe due to lack of easy availability of GF foods outside the home or peer pressure when dining out, as in other studies [12, 18, 19, 21]. In the Ljungman and Myrdal study [27], they stratified the adolescents' ages and observed that the adherence was better in younger adolescents aged 12 to 14 years old than in older adolescents aged 15 to 17 years old, probably because the latter group had greater social exposure than the former.

In our study the majority (93.8\%) of patients reported that they had suffered from symptoms of the disease for about 23.6 months before diagnosis (DP: 24 months), similar to the time span reported in another study [12], and longer than in others $[17,18,22,26]$.

In our series, most of the children had typical symptoms of CD (88.6\%), which was consistent with the study by Charalampopoulos et al. [12] but higher than in other studies [17, 18, 22, 26]. Our findings also contrast with those of Munoz et al. [24], who reported that children with atypical symptoms before diagnosis and patients with screening diagnosis by serologic testing had a higher rate of transgression than children with typical symptoms.

Other studies reported similar reasons, regarding to the availability of GF foods and costs as the most significant barriers to adherence to a GFD [24, 28].

In contrast as described previously [18], in our study there was not an association between non-adherence and environment. Our study also agree with others studies $[19,29]$ that most CD patients on a GFD, specially teenagers, found it easier to adhere to the diet at home and school than when out in social situations. Our study found that the most transgressions did not occur only at friends' house and birthday parties, but also at home and school, as reported by Munoz et al. [24].

In our patients, as well as in other study [30], when asked about the behavior at parties and social events,

Table 4 Nutritional status of patients with celiac disease according to the body mass index for age (BMI/age)

\begin{tabular}{llllll}
\hline & At time of diagnosis & 1 year & 2 years & 5 years & Current \\
\hline Extremely underweight & 2 & 0 & 0 & 1 & 0 \\
Underweight & 2 & 0 & 8 & 9 & 0 \\
Eutrophy & 18 & 16 & 3 & 0 & 19 \\
Risk of overweight & 2 & 4 & 2 & 0 & 2 \\
Overweight & 2 & 3 & 2 & 11 & 2 \\
Obesity & 1 & 2 & 16 & 27 \\
Total & 27 & 25 & & \\
\hline
\end{tabular}


Table 5 Stature for age of patients with celiac disease at diagnosis and over time

\begin{tabular}{|c|c|c|c|c|c|}
\hline & At time of diagnosis & 1 year & 2 years & 5 years & Current \\
\hline Very short stature for age & 4 & 3 & 2 & 1 & 1 \\
\hline Short stature for age & 5 & 4 & 2 & 1 & 3 \\
\hline Appropriate stature for age & 18 & 18 & 12 & 9 & 22 \\
\hline Total & 27 & 25 & 16 & 11 & 26 \\
\hline
\end{tabular}

the majority reported that successful diet adherence strategies included planning ahead and taking their own food to social events to avoid meals containing gluten.

Most patients in our study found the GFD relatively easy to follow, were comfortable talking about their disease and realized that they would need to follow a GFD for entire life. This results differs from that reported by Bravo \& Munoz [24] reported that 70\% found the diet hard to follow, $55 \%$ had difficulty following the diet, $42.8 \%$ were not sure what they could eat, and $51.5 \%$ reported that the GFD was causing financial distress in the family.

In respect of involuntary transgression, it is worth mentioning the possibility of occurring with the acquisition of cheese bread outside the home (cited by nine patients) as this may be contaminated with other gluten foods produced and served alongside the bread. In addition, despite reporting adherence to the diet, the positive EMAs results in 28/35 patients indicate unintentional transgression.

Error or mistake was also cited in the literature as a reason for transgressions. In Brazil the law requires that all processed products show the information "contains" or "does not contain gluten", and even with disbelief in some information on the label, it facilitates the purchase of industrial products. However, errors or mistakes often happen with ready-to-eat products, consumed in cafes, restaurants and while traveling, so it is important that patients and families are able to cope with these situations. In this study, $48.1 \%$ of patients report that the transgression was not intentional, but rather due to the lack of adequate information about the preparation of food.

Similar to our results, several studies have found that patients found it particularly difficult to adhere to a GFD when dining out or at social events, due to variability in the availability of GFD foods or crosscontamination with gluten.

As our study, the higher price of gluten free products is a point of great concern, and several studies from North America and Europe have demonstrated that the cost of GFD food products is significantly higher than that of gluten-containing foods [31-33].

Similar to our results, Tapsas et al. [20] reported different symptoms after incidental intake of gluten, including abdominal pain (64.8\%), vomiting (33.3\%), and diarrhea
(24.1\%). They found that in two-thirds of the children in their study, symptoms were noticed soon after consumption, that is, during the first $3 \mathrm{~h}$. No correlation was found between the amount of accidental gluten intake and the time of symptom onset. Some patients, however, experienced symptoms later, that is, after more than $24 \mathrm{~h}$.

Dietary adherence has been the subject of several investigations in pediatric $\mathrm{CD}$ and these studies have varied with respect to sample size and method of assessment of adherence. Measures used to assess adherence included self-report, interview, dietary record, and biossay methods, and each measure has its strengths and limitations. Available methods are insufficiently accurate to identify occasional gluten exposure that may cause intestinal mucosal damage. Serological tests are highly sensitive and specific for diagnosis, but do not predict recovery and are not useful for follow-up. Serial endoscopy is invasive and impractical for frequent monitoring, and dietary interview can be subjective [10]. The detection of gluten immunogenic peptides in feces and urine as new non-invasive biomarkers of gluten intake has been suggested, but requires further investigation [34].

In our study, the overall rate of adherence was $80 \%$, as measured by questionnaire and serologic testing. Another Brazilian study conducted by ACELBRA (Brazilian Celiac Association) [35] found that 29.5\% of patients reported transgressions in their diet, a higher percentage than the present study. The rates of adherence to GFD in the literature vary from $39 \%$ to $79 \%$, depending on factors including self-reporting, laboratory testing and prediagnosis symptoms [12, 18, 19, 36-39]. Some of the studies, despite the small sample, illustrated that nonadherence to GFD is related to poorer bone health [36, 37], shorter stature [37]. Parent report adherence via interview was higher than bioassay data [38] tTG antibodies were significantly more sensitive at identifying occasional non-adherence than EMAs data [39]. Younger age at diagnosis, currently being a teenager, and current symptoms were associated with nonadherence [18]. There was a relationship between compliance, children's age and perceived parental knowledge [12]. Younger age at diagnosis and shorter duration since the diagnosis were associated with a better adherence rate [22]. 
Our study did not compare the potential difference in diet adherence when questionnaires were filled in by adolescents or by their parents, or if the $\mathrm{CD}$ education program for parents and caregivers of celiac patients improved adherence to GFD rate. In our service all the family and responsible for celiac patients receive education about $\mathrm{CD}$ routinely.

It has been reported that parents could give us a false picture of the children's compliance with the GFD [12, 40] On the other hand, other authors reported that the perceived parental knowledge was also independently and significantly associated with dietary compliance [12].

We would like to strengthen the importance of the education on CD and the adherence of a GFD not only to the pediatric patients but also to the family and all those involved with the patient to achieve the adherence to a strict lifelong gluten-free diet (GFD).

In our study, children and adolescents recovered their nutritional status after starting treatment, with a significant increase in the proportion of overweight patients over the years. There was no statistical relationship between adherence to treatment and nutritional status, which result was in agreement with other study [41]. They also reported that children who did not adhere to the GFD had lower values of the Z-score of stature/age in comparison with children who adhered to the GFD; $5.9 \%$ of children were overweight, which was lower than found in our study.

Other study which reported nutritional status [26], found that almost $19 \%$ of patients had an elevated BMI at diagnosis (12.6\% overweight, $6 \%$ obese) and $74.5 \%$ presented with a normal BMI. Seventy-five percent of patients with an elevated BMI at diagnosis decreased their BMI Z-score significantly after adherence to a GFD, normalizing it in $44 \%$ of cases. Of patients with a normal BMI at diagnosis, weight Z-scores increased significantly after treatment, and $13 \%$ became overweight. In this study, a GFD may have a beneficial effect upon the BMI of overweight and obese children with $\mathrm{CD}$. Other aspect about nutrition and $C D$ was observed in other study [25] which reported that children with $C D$ had high intakes of fiber, and foods with a high glycemic index, and glycemic load, and lower folate content than controls. This has implications for dietary counseling in this population.

The limitations of our study were the sample size and the lack of complete data regarding nutritional evolution, which may have interfered in the absence of association between transgression and other variables, including nutritional status. The retrospective analysis of medical records and questionnaires may involve registration and memory bias, respectively, which may influence the accuracy of the results.

\section{Conclusion}

In this study, a fifth of the patients was voluntarily transgressing the GFD, mainly because of the high cost, lack of availability and poor palatability related to the diet. Possibly, part of the children in sample is also involuntarily eating gluten, as observed by the positivity of the serologic tests. There was a catch-up growth in patients with nutritional deficit and a risk of excessive weight gain during follow-up. The early diagnosis and continuous education, with a multidisciplinary clinical care model, is essential to promote adherence to the diet.

\section{Additional file}

Additional file 1: Questionnaire for assessment of adherence to the treatment of patients with Celiac Disease. Questionnaire constructed for this study comprising two open questions on social events and difficulties with the GFD, and closed questions about food routine, awareness, adherence and difficulties regarding the GFD. (DOC $75 \mathrm{~kb}$ )

\section{Abbreviations}

ACELBRA: Brazilian Celiac Association; BMI: Body mass index; CAPPesq: Ethics Committee for review of research projects; CD: Celiac disease; EMAs: Antiendomysial antibodies; GFD: Gluten-free diet; HLA: Human histocompatibility antigen genes; REC: Research Ethics Committee; SMUSP: School of Medicine, University of Sao Paulo; SPSS: Statistical Package for the Social Sciences; TG2IgA: Transglutaminase antibodies 2; WHO: World Health Organization

\section{Acknowledgements}

We are grateful to all the patients who participated in this study. All the authors have read this manuscript and approved the submission for publication. All authors fulfill the criteria given in the authorship defined by your journal. All author concor with the submittion and none of the data have been previously reported or are under consideration for publication elsewhere.

No conflict of interest exists for any of the authors.

Funding

No funding was recieved.

Availability of data and materials

The datasets during and/or analysed during the current study available from the corresponding author on reasonable request.

\section{Authors' contributions}

MR: coordinated and designed the study, participated in analysis and interpretation of data, wrote and finalized the manuscript. CAS: participated in the collection, writing and interpretation of data. GHY: participated in the collection, writing and interpretation of data. All authors read and approved the final version of the manuscript.

\section{Ethics approval and consent to participate \\ This Project was approved by the Research Ethics Committee (REC) at Department of Pediatrics, Hospital das Clinicas, São Paulo, Brazil, and Ethics Committee for review of research projects (CAPPesp) under the number $829 / 11$. Permission to conduct the study was sought from relevant ethical committees at Department of Pediatric, Hospital das Clinicas, São Paulo, Brazil. All patients were entered into the study after a written informed consent, either given by the patients themselves or their guardians in the case where patients were not able to.}

\section{Consent for publication}

Not applicable.

\section{Competing interests}

The authors declare that they have no competing interests. 


\section{Publisher's Note}

Springer Nature remains neutral with regard to jurisdictional claims in published maps and institutional affiliations.

\section{Author details}

'Department of Gastroenterology, Hospital das Clínicas, School of Medicine, University of Sao Paulo (SMUSP), Av. Dr Eneas de Carvalho Aguiar, 255, 05403-000, Sao Paulo, Brazil. ²Department of Pediatric, Instituto da Criança, Division of Nutrition, Hospital das Clínicas, School of Medicine, University of Sao Paulo (SMUSP), Sao Paulo, Brazil.

Received: 14 June 2017 Accepted: 8 January 2018

Published online: 19 January 2018

\section{References}

1. Fasano A, Araya M, Bhatnagar S, Cameron D, Catassi C, Dirks M, Mearin ML, Ortigosa L, Phillips A; Celiac Disease Working Group, FISPGHAN. Federation Of international societies of pediatric gastroenterology, hepatology, and nutrition consensus report on celiac disease. J Pediatr Gastroenterol Nutr 2008:47(2):214-219.

2. Leffler DA, Green PHR, Fasano A. Extraintestinal manifestations of coeliac disease. Nat Rev Gastroenterol Hepatol. 2015;12:561-71.

3. Catassi C, Gatti S, Lionetti E. World perspective and celiac disease epidemiology. Dig Dis. 2015;33(2):141-6.

4. Oliveira RP, Sdepanian VL, Barreto JA, Cortez AJ, Carvalho FO, Bordin JO, de Camargo Soares MA, da Silva Patrício FR, Kawakami E, de Morais MB, Fagundes-Neto U. High prevalence of celiac disease in Brazilian blood donor volunteers based on screening by lgA antitissue transglutaminase antibody. Eur J Gastroenterol Hepatol. 2007;19(1):43-9.

5. Alencar ML, Ortiz-Agostinho CL, Nishitokukado L, Damião AO, AbrantesLemos CP, Leite AZ, Td B, Chamone Dde A, Silva ME, Giannella-Neto D, Sipahi AM. Prevalence of celiac disease among blood donors in São Paulo: the most populated city in Brazil. Clinics (Sao Paulo). 2012;67(9):1013-8.

6. Melo SB, Fernandes MI, Peres LC, Troncon LE, Galvão LC. Prevalence and demographic characteristics of celiac disease among blood donors in Ribeirão Preto, state of São Paulo, Brazil. Dig Dis Sci. 2006;51(5):1020-5.

7. Pereira MA, Ortiz-Agostinho CL, Nishitokukado I, Sato MN, Damião AO, Alencar ML, Abrantes-Lemos CP, Cançado EL, de Brito T, loshii SO, Valarini SB, Sipahi AM. Prevalence of celiac disease in an urban area of Brazil with predominantly European ancestry. World J Gastroenterol. 2006, 28;12(40):6546-50.

8. Gandolfi L, Pratesi R, Cordoba JC, Tauil PL, Gasparin M, Catassi C. Prevalence of celiac disease among blood donors in Brazil. Am J Gastroenterol. 2000; 95(3):689-92.

9. Garnier-Lengliné H, Cerf-Bensussan N, Ruemmele FM. Celiac disease in children. Clin Res Hepatol Gastroenterol. 2015;39(5):544-51.

10. Hommel KA, Mackner LM, Denson LA, Crandall W. Treatment regimen adherence in pediatric gastroenterology. J Pediatr Gastroenterol Nutr. 2008; 47(5):526-43.

11. Zarkadas M, Dubois S, Maclsaac K, Cantin I, Rashid M, Roberts KC, La Vieille S, Godefroy S, Pulido OM. Living with coeliac disease and a gluten-free diet: a Canadian perspective. J Hum Nutr Diet. 2013;26(1):10-23.

12. Charalampopoulos D, Panayiotou J, Chouliaras G, Zellos A, Kyritsi E, Roma E. Determinants of adherence to gluten-free diet in Greek children with coeliac disease: a cross-sectional study. Eur J Clin Nutr. 2013;67(6):615-9.

13. Valitutti F, Trovato CM, Montuori M, Cucchiara S. Pediatric Celiac Disease: Follow-Up in the Spotlight. Adv Nutr. 2017, 15;8(2):356-361.

14. Husby S, Koletzko S, Korponay-Szabó IR, Mearin ML, Phillips A, Shamir R, Troncone R, Giersiepen K, Branski D, Catassi C, Lelgeman M, Mäki M, RibesKoninckx C, Ventura A, Zimmer KP; ESPGHAN working group on coeliac disease diagnosis.; ESPGHAN gastroenterology committee.; European Society for Pediatric Gastroenterology, hepatology, and nutrition. European Society for Pediatric Gastroenterology, Hepatology, and Nutrition guidelines for the diagnosis of coeliac disease.J Pediatr Gastroenterol Nutr 2012;54(1):136-160.

15. Oberhuber $G$, Granditsch $G$, Vogelsang $H$. The histopathology of coelic disease: time for a standardized report scheme for pathologists. Eur J Gastroenterol Hepatol. 1999;11:1185-94.

16. WHO Multicentre Growth Reference Study Group. WHO child growth standards: length/height-for-age, weight-for-age, weight-for-length, weightfor-height and body mass index-for-age: methods and development. Geneva: World Health Organization; 2006.
17. Mozer-Glassberg Y, Zevit N, Rosenbach Y, Hartman C, Morgenstern S, Shamir R. Follow-up of children with celiac disease - lost in translation? Digestion. 2011;83(4):283-7.

18. Kurppa K, Lauronen O, Collin P, Ukkola A, Laurila K, Huhtala H, Mäki M, Kaukinen K. Factors associated with dietary adherence in celiac disease: a nationwide study. Digestion. 2012;86(4):309-14.

19. MacCulloch K, Rashid M. Factors affecting adherence to a gluten-free diet in children with celiac disease. Paediatr Child Health. 2014;19(6):305-9.

20. Tapsas D, Fälth-Magnusson K, Högberg L, Hammersjö JÅ, Hollén E. Swedish children with celiac disease comply well with a gluten-free diet, and most include oats without reporting any adverse effects: a long-term follow-up study. Nutr Res. 2014;34(5):436-41.

21. Barnea L, Mozer-Glassberg Y, Hojsak I, Hartman C, Shamir R. Pediatric celiac disease patients who are lost to follow-up have a poorly controlled disease. Digestion. 2014;90(4):248-53.

22. Sarkhy AA, El Mouzan Ml, Saeed E, Alanazi A, Alghamdi S, Anil S, Assiri A. Clinical Characteristics of Celiac Disease and Dietary Adherence to Gluten-Free Diet among Saudi Children. Pediatr Gastroenterol Hepatol Nutr. 2015;18(1):23-9.

23. Webb C, Myléus A, Norström F, Hammarroth S, Högberg L, Lagerqvist C, Rosén A, Sandström O, Stenhammar L, Ivarsson A, Carlsson A. High adherence to a gluten-free diet in adolescents with screening-detected celiac disease. J Pediatr Gastroenterol Nutr. 2015:60(1):54-9.

24. Bravo MF, Munõz FMP. Adherence and impact of gluten free diet in children with celiac disease. Rev Chil Pediatr. 2011;82(3):191-7.

25. Alzaben AS, Turner J, Shirton L, Samuel TM, Persad R, Mager D. Assessing nutritional quality and adherence to the gluten-free diet in children and adolescents with celiac disease. Can J Diet Pract Res. 2015;76(2):56-63.

26. Reilly NR, Aguilar K, Hassid BG, Cheng J, Defelice AR, Kazlow P, Bhagat G, Green PH. Celiac disease in normal-weight and overweight children: clinical features and growth outcomes following a gluten-free diet. J Pediatr Gastroenterol Nutr. 2011;53(5):528-31.

27. Ljungman G, Myrdal U. Compliance in teenagers with coeliac disease-a Swedish follow-up study. Acta Paediatr. 1993:82(3):235-8.

28. Rashid M, Cranney A, Zarkadas M, Graham ID, Switzer C, Case S, Molloy M, Warren RE, Burrows V, Butzner JD. Celiac disease: evaluation of the diagnosis and dietary compliance in Canadian children. Pediatrics. 2005;116(6):e754-9.

29. Olsson C, Lyon P, Hörnell A, Ivarsson A, Sydner YM. Food that makes you different: the stigma experienced by adolescents with celiac disease. Qual Health Res. 2009;19(7):976-84.

30. Bacigalupe G, Plocha A. Celiac is a social disease: family challenges and strategies. Fam Syst Health. 2015;33(1):46-54.

31. Stevens L, Rashid M. Gluten-free and regular foods: a cost comparison. Can J Diet Pract Res. 2008 Fall;69(3):147-50.

32. Lee AR, Ng DL, Zivin J, Green PH. Economic burden of a gluten-free diet. J Hum Nutr Diet. 2007;20(5):423-30.

33. Singh J, Whelan K. Limited availability and higher cost of gluten-free foods. J Hum Nutr Diet. 2011;24(5):479-86.

34. Moreno ML, Rodríguez-Herrera A, Sousa C, Comino I. Biomarkers to Monitor Gluten-Free Diet Compliance in Celiac Patients. Nutrients. 2017, 6;9(1), pii: E46..

35. Sdepanian VL, de Morais MB, Fagundes-Neto U. [Celiac disease: evaluation of compliance to gluten-free diet and knowledge of disease in patients registered at the Brazilian Celiac Association (ACA)]. Arq Gastroenterol 2001; 38(4):232-239.

36. Mora S, Barera G, Beccio S, et al. A prospective, longitudinal study of long term effect of treatment on bone density in children with celiac disease. J Pediatr. 2001;139:516-21.

37. Hartman C, Hino B, Lerner A, et al. Bone quantitative ultrasound and boné mineral density in children with celiac disease. JPediatr Gastroenteorl Nutr. 2004;39:504-10.

38. Tommasini A, Not T, Kiren V, et al. Mass screening for coelic disease using antihuman transglutaminase antibody assay. Arch Dis Child. 2004:89:512-5.

39. Bazzigaluppi E, Roggero P, Parma B, et al. Antibodies to recombinant human tissue-transglutaminase in coeliac disease: diagnostic effectiveness and decline pattern after gluten-free diet. Dig Liver Dis. 2006;38:98-102.

40. Byströn IM, Hollen E, Fälth-Magnusson K, Johansson A. Health-related quality of life in children and adolescents with celiac disease: from the perspectives of children and parents. Gastroenterol Res Pract. 2012;2012(986475)

41. Andreoli CS, Cortez APB, Sdepanian VL, Morais MB. Nutritional evaluation and food intake of celiac disease patients compliant or not with a glutenfree diet. Rev Nutr. Campinas. 2013:26(3):301-11. 